\title{
Effect of Silicon Content on Corrosion Resistance of a Cold Rolled Non-Oriented Silicon Steel
}

\author{
Yuan Lin $^{1,2, *}$, Hongxia Wang ${ }^{1, *}$, Jin Xiang $^{3}$, Hui Wei ${ }^{1}$, Zilong Zhao ${ }^{4, *}$, Wenkang Zhang ${ }^{2}$, Yide Wang ${ }^{1,2}$ \\ ${ }^{1}$ College of Materials Science and Engineering, Taiyuan University of Technology, Taiyuan 030024, \\ China \\ ${ }^{2}$ Technical Center, Taiyuan Iron \& Steel (Group) Co., Ltd., Taiyuan 030003, China. \\ ${ }^{3}$ Department of Mechanics, Jinzhong University, Jinzhong, 030600, Shanxi, China \\ ${ }^{4}$ School of Chemical Engineering and Technology, Sun Yat-sen University, Zhuhai 519082, China \\ *E-mail: linyuan@ tisco.com.cn; wanghxia1217@163.com; zhaozlong@ mail.sysu.edu.cn
}

Received: 3 December 2021 / Accepted: 24 January 2022 / Published: 2 February 2022

\begin{abstract}
Non-oriented silicon steel is a critical functional material for various types of motor iron cores. When the motor was rotating at high speed in the rainy season, the iron core materials rusted easily. In this paper, the corrosion resistance of low silicon, medium silicon and high silicon non-oriented silicon steel in wet and hot environments was studied. The phase of three materials was tested by XRD, the microstructure and surface morphology before and after corrosion of silicon steel were studied and compared by three-dimensional ultradepth-of-field microscopy. The morphology and composition after corrosion of silicon steel were measured by scanning electron microscope and composition analysis spectrum. The corrosion mechanism of cold rolled non-oriented silicon steel with different silicon contents in wet and hot extreme environments was discussed.
\end{abstract}

Keywords: non-oriented silicon steel, Si content, corrosion resistance, oxidization film

\section{$\underline{\text { FULL TEXT }}$}

(C) 2022 The Authors. Published by ESG (www.electrochemsci.org). This article is an open access article distributed under the terms and conditions of the Creative Commons Attribution license (http://creativecommons.org/licenses/by/4.0/). 